\title{
RUNX1 Mutations Can Lead to Aberrant Expression of CD79a and PAX5 in Acute Myelogenous Leukemias: A Potential Diagnostic Pitfall
}

\author{
Thomas Menter $^{\mathrm{a}}$ Pontus Lundberg ${ }^{\mathrm{b}} \quad$ Friedel Wenzel $^{\mathrm{a}} \quad$ Jan Dirks $^{\mathrm{b}} \quad$ Paula Fernandez $^{\mathrm{c}}$ \\ Dorothea Friess $^{d}$ Stefan Dirnhofer ${ }^{a} \quad$ Alexandar Tzankov $^{a}$ \\ anstitute of Pathology and Medical Genetics, University Hospital Basel, Basel, Switzerland; b Department of \\ Hematology, University Hospital Basel, Basel, Switzerland; ' Department of Laboratory Medicine, Cantonal Hospital \\ Aarau, Aarau, Switzerland; dDepartment of Hematology, Cantonal Hospital Olten, Olten, Switzerland
}

\section{Established Facts}

- Acute myelogenous leukemia (AML) with RUNX1-RUNX1T1 fusion frequently aberrantly expresses the B cell antigens CD19, PAX5, and CD79a. This is due to net inactivation of the myeloid master regulator PU.1 by the chimeric RUNX1-RUNX1T1 protein.

- AML with the RUNX1 mutation is a provisional AML sub-entity in the WHO 2017 classification. Such mutations are commonly observed in mixed-lineage leukemia (MLL), and in FLT3-ITD and +13 and +21 AML.

\section{Novel Insights}

- RUNX1 mutant AML can aberrantly express the B cell antigens CD79a and/or PAX5, similarly to AML with RUNX1-RUNX1T1 fusion.

- CD79a and PAX5 expression combined with myeloperoxidase negativity or mere detectablity in RUNX1 mutant AML is a potential classificatory pitfall that may lead to misclassification of respective cases as acute lymphoblastic leukemia (ALL).

- The expression of B cell markers in RUNX1 mutant AML may be linked to deregulation of the myeloid master regulator PU.1, similarly to what is known for AML with RUNX1-RUNX1T1 fusion.

\section{Keywords}

RUNX1 - CD79a - PAX5 - Acute myelogenous leukemia ·

Acute lymphoblastic leukemia

\section{Abstract \\ Background: RUNX1 is a crucial transcription factor for he- matological stem cells and well-known for its association with acute lymphoblastic leukemia (ALL) and acute myelog-}

enous leukemia (AML). Besides the translocation $t(8 ; 21)$ that leads to the RUNX1-RUNX1T1 fusion, somatic mutations of RUNX1 have been discovered. Methods: Four bone marrow trephine biopsies of patients with CD79a-positive and/or PAX5-positive acute leukemias were investigated by immunohistochemistry (IHC), karyotyping, and next-generation sequencing-based genetic analysis. Data were then compared to a historical collective of AML $(n=42)$ and 42 cases of AML newly diagnosed at our institution between June
KARGER

(c) 2018 S. Karger AG, Basel

E-Mail karger@karger.com

www.karger.com/pat
Prof. Dr. med. Alexandar Tzankov

Institute of Pathology

Schönbeinstrasse 40

$\mathrm{CH}-4031$ Basel (Switzerland)

E-Mail alexandar.tzankov@usb.ch 
2017 and May 2018. Results: We report on 4 cases of acute leukemia with an equivocal immunophenotype showing expression of CD79a and/or PAX5, which led to a preliminary histopathologic classification as probable ALL/unclassifiable acute leukemia. All cases were positive for CD34 and TdT but negative for several myeloid markers on IHC. Mutational analysis revealed point mutations and indels of $R U N X 1$ and further mutations typical for AML such as TET2, DNMT3A, and SRSF2, and 2 cases had tetrasomy 13 characteristic of RUNX1 mutant AML. Conclusion: Aberrant CD79a and/or PAX5 expression can be found in AML cases with RUNX1 mutations even without the translocation $t(8 ; 21)$. Our series shows the expression of CD79a and PAX5 to be a potential pitfall in the classification of RUNX1 mutant acute leukemia.

(c) 2018 S. Karger AG, Basel

Runt-related transcription factor 1 (RUNX1) (formerly known as acute myeloid leukemia 1 protein [AML1]) is crucial for hematopoietic stem cells [1] and plays an oncogenic role in both acute lymphoblastic leukemia (ALL) and acute myelogenous leukemia (AML) [2]. The best-studied aberration of RUNX1 in AML is $\mathrm{t}(8 ; 21)$ (q22;q22), generating the fusion protein RUNX1RUNX1T1 (formerly described as AML1-ETO), resulting in a loss of function/repression of normal RUNX1 [3]. This translocation and $\mathrm{t}(12 ; 21)(\mathrm{p} 13 ; \mathrm{q} 22)$ in ALL are considered to be initiating events in leukemogenesis [4]. AML with $t(8 ; 21)$ can aberrantly express $B$ cell markers, especially CD19, CD79a, and PAX5 [5, 6].

Besides $\mathrm{t}(8 ; 21)$, different types of RUNX1 somatic mutations have been more recently discovered in myeloid malignancies [2]. They all result in a loss of function of RUNX1 and mainly affect the Runt homology domain, which is responsible for the DNA-binding and transcriptional activity of RUNX1 [7]. In contrast to AML with $t(8$; 21 ), these cases are associated with a worse prognosis, referred to by the WHO as a provisional entity "AML with mutated RUNX1" [8]. The negative impact of RUNX1 mutations has also been very recently confirmed in AML in children [9]. Contrary to $\mathrm{t}(8 ; 21)(\mathrm{q} 22 ; \mathrm{q} 22)$, RUNX1 mutations are rarely discovered as initiating mutations in AML, and are therefore considered secondary events driving the progression of the disease [10]. They are also rare in clonal cytopenia of undetermined significance (CCUS) and overt myelodysplastic syndromes and have not yet been observed in clonal hematopoiesis of indeterminate potential (CHIP) [4]. RUNX1 germline mutations are associated with "familial platelet disorder with predisposition to AML" (FPD/AML), and implicate a $44 \%$ life-

RUNX1 Mutations and Aberrant

Expression of CD79a and PAX5 in AML time risk of developing myelodysplastic syndrome or AML [11], but they usually also affect domains other than the Runt homology domain.

We report on a case series of 4 patients with CD79apositive and/or PAX5-positive, difficult-to-classify AML, with mutated RUNX1 (Table 1). Cases were investigated by immunohistochemistry (IHC), karyotyping, and nextgeneration sequencing (NGS)-based panel sequencing, and then compared to a historical collective of AML $(n=$ 42) stained for the B cell markers CD19, CD20, CD79a, and PAX5 [12] as well as 42 AML cases newly diagnosed in routine practice at our institution between June 2017 and April 2018.

These 4 cases with an equivocal immunophenotype, sharing negativity for myeloperoxidase (MPO; $3 / 4$ cases determined on IHC), CD19, and CD20, but expressing CD79a (clone SP18) and/or PAX5 (clone SP34), were preliminary histopathologically classified as probable ALL or unclassifiable acute leukemia, most probably AML or mixed-phenotype acute leukemia (MPAL). All were positive for $\mathrm{CD} 34$ and $\mathrm{TdT}$, all (but one) were negative for the additional myeloid marker $\mathrm{CD} 11 \mathrm{c}$, and all were negative for CD14 and CD117 on IHC (Fig. 1). On flow cytometry (FCM), the blasts of all cases were positive for the myeloid markers CD33, CD13 ( \pm ), CD117 ( \pm and $\operatorname{dim})$, and CD15 (2/4 cases), and negative for CD19, CD20, CD10, CD22, and cCD79a. FCM thus favored AML (Table 1).

NGS analysis was performed on bone marrow DNA, isolated using QIAsymphony (QIAGEN, Hilden, Germany) according to the manufacturer's protocol. Patient NGS libraries were prepared using the AML community panel from ThermoFisher (Waltham, MA, USA) containing 19 genes frequently mutated in AML (www. ampliseq.com), sequenced using the Ion S5 platform, and analyzed on the Ion Reporter AML pipeline (ThermoFisher). The average coverage per sample was $>3,000$. Sensitivity for calling a mutation was set at 3\%. Mutational analysis revealed deleterious (frameshift and nonsense) or expected deleterious double or single mutations of RUNX1, i.e., G165fs and E86fs (with a variant allelic frequency [VAF] of 46 and 48\%, respectively), R166* (VAF of 14\%), R166Q and D160E (VAF of 15 and 18\%, respectively), and $\mathrm{W} 106 \mathrm{C}$ (VAF of $86 \%$ ), with a pathogenic score of at least 0.86 . The VAF of mutant RUNX1 in the last 3 patients strongly argued against germline mutations. While bearing RUNX1 mutations with a VAF close to $50 \%$, the first patient presented with AML at the age of 84 years and had no history of hematological illness, particularly not of an inherited bleeding disorder, 
Table 1. Characteristics of 4 cases

\begin{tabular}{|c|c|c|c|c|}
\hline & Patient \#1 & Patient \#2 & Patient \#3 & Patient \#4 \\
\hline Age at presentation & 84 & 63 & 48 & 74 \\
\hline Sex & male & male & female & male \\
\hline Chemotherapy regimen & 1 cycle of azacitidine & $\begin{array}{l}\text { induction therapy } \\
\text { (idarubicin and cytarabine) }\end{array}$ & $\begin{array}{l}\text { HOVON } 132 \\
\text { (lenalidomide arm) }\end{array}$ & azacitidine \\
\hline HCT & none & allogenic & allogenic & none \\
\hline \multicolumn{5}{|l|}{ Immunohistochemistry } \\
\hline Positive markers & $\begin{array}{l}\text { CD34, CD79a, CD123 } \pm \\
\text { PAX5, TdT }\end{array}$ & CD34, CD79a, LMO2, TdT & $\begin{array}{l}\text { CD11c dim, CD34, CD79a, } \\
\text { CD117 dim, CD123 } \\
\text { CD303 }, \text { TdT }\end{array}$ & $\begin{array}{l}\text { CD11c, CD34, CD79a, } \\
\text { CD123 } \pm, \text { MPO } \pm \text {, PAX5, } \\
\text { TdT }\end{array}$ \\
\hline \multicolumn{5}{|l|}{ Flow cytometry } \\
\hline Positive markers & 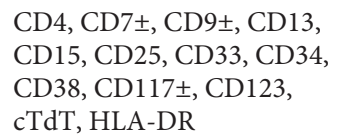 & $\begin{array}{l}\text { CD7, CD11b } \pm, C D 33, C D 34 \\
\text { CD38, CD71 } \\
\text { CD123, cD } 117 \pm\end{array}$ & 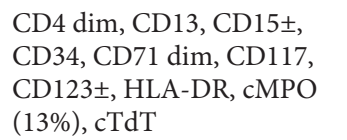 & $\begin{array}{l}\text { CD7 } \pm \text {, CD13, CD 34, } \\
\text { CD38, CD71, CD117, } \\
\text { CD123, cTdT, HLA-DR }\end{array}$ \\
\hline Negative markers & $\begin{array}{l}\text { CD3, CD9, CD10, CD11b, } \\
\text { CD14, CD16, CD19, CD21, } \\
\text { CD35, CD36, CD41, CD42a, } \\
\text { CD56, CD58, CD64, CD79a, } \\
\text { CD105, cMPO, CD203, } \\
\text { CD300e }\end{array}$ & $\begin{array}{l}\text { CD1a, CD2, cCD3, CD3, CD4, } \\
\text { CD5, CD8, CD9, CD10, CD13, } \\
\text { CD14, CD15, CD16, CD19, } \\
\text { CD22, CD25, CD35, CD36, } \\
\text { CD41, CD42a, CD42b, CD 56, } \\
\text { CD61, CD64, cCD79a, CD105, } \\
\text { CD203c, CD300e, HLA-DR, } \\
\text { cMPO, NG2 }\end{array}$ & $\begin{array}{l}\text { CD2, cCD3, sCD3, CD7, } \\
\text { CD10, CD14, CD16, CD19, } \\
\text { CD33, CD35, CD36, CD64, } \\
\text { cCD79a, CD79b, CD56, } \\
\text { CD300e, lactoferrin, lyso- } \\
\text { zyme }\end{array}$ & $\begin{array}{l}\text { CD3, CD 4, CD10, } \\
\text { CD11b, CD14, CD15, } \\
\text { CD16, CD19, CD20, } \\
\text { CD21, CD22, CD33, } \\
\text { CD35, CD36, CD 56, } \\
\text { CD64, CD79a, CD105, } \\
\text { CD203, CD300e, cMPO }\end{array}$ \\
\hline
\end{tabular}

Bold type denotes B cell markers aberrantly expressed on AML blasts. AML, acute myelogenous leukemia; MPO, myeloperoxidase; FAB, FrenchAmerican-British; HCT, hematopoietic cell transplantation; NGS, next-generation sequencing.

and G165fs was near to the well-known R166 mutational hot-spot in AML, all of which argued against germline mutations of RUNX1. Indeed, there are no germline variants reported at codon 165 or 166 that would be compatible with the mutations observed in this study. In FPD/ AML, $>40$ different RUNX1 mutations are described and show varied distribution at many different sites [13], but they are expected to occur with a VAF near to 50 or $100 \%$. Additional prototypic mutations of AML such as DNMT3A, NRAS, and SRSF2 in 2 patients, and PTPN11 and TET2 in 1 patient each, respectively, were detected (Table 1); these mutations as well as the tetrasomy 13 of cases 1 and 4 being prototypic for RUNX1-mutated AML subcohorts of larger studies [14]. All cases were devoid of $t(8: 21)$. In our previously studied collective of $42 \mathrm{AML}$ cases [12], only 2 cases showed an expression of CD19 and PAX5, both of them harboring $\mathrm{t}(8 ; 21)$. Neither CD79a-positive nor PAX5-positive cases were detected in the 42 RUNX1 mutation- and $\mathrm{t}(8 ; 21)$-negative newly diagnosed AML cases in our routine practice between June 2017 and May 2018.

Expression of CD19, CD79a, and PAX5 in AML with $t(8 ; 21)$ is well documented in the literature $[5,6]$. With the case series presented here, we show that aberrant CD79a and PAX5 expression can also be recurrently found in AML with RUNX1 mutations in the absence of 


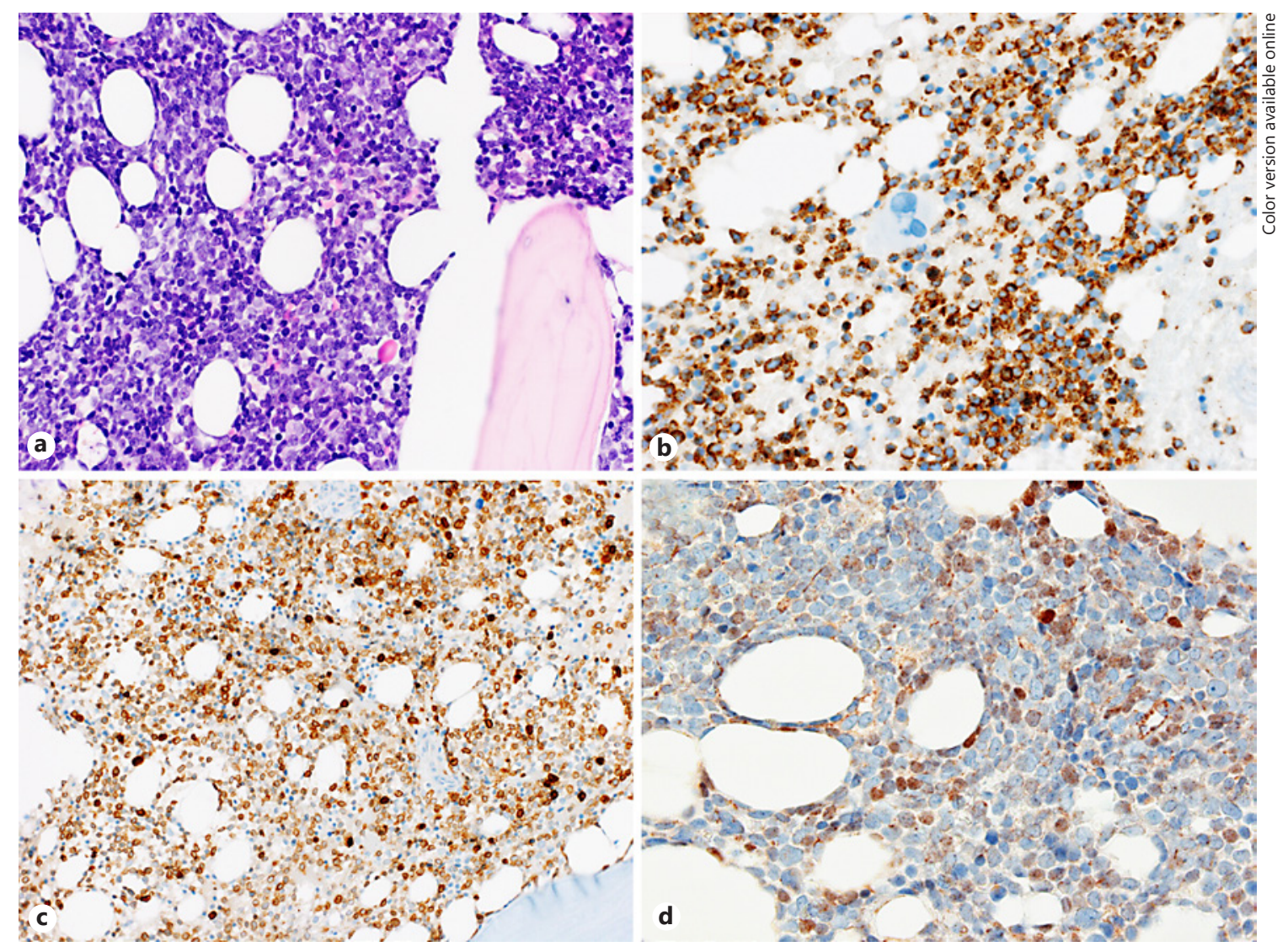

Fig. 1. a Morphology showing a diffuse infiltrate of blastoid cells resembling lymphoid rather than myeloid blasts. HE. $\times 200$. The blastoid cells show expression of CD34 (b, $\times 200)$, CD79a $(\mathbf{c}, \times 200$; clone SP18), and focally PAX5 (d, $\times 360$; clone SP34).

$\mathrm{t}(8 ; 21)$. The reason why CD79a was not detected by FCM could have been the use of a different clone (HM57), which binds to an epitope different from what the clone SP18 used for IHC binds (SP18: epitope at the N-terminal extracellular region of CD79a; HM57: epitope within the cytoplasmic domain of CD79a).

Importantly, translocations and mutations lead to a loss of function of RUNX1, thus postulating a protective, antileukemogenic effect of functional RUNX1. The leukemogenic effect of RUNX1 alterations expands beyond its role as a transcription factor; RUNX1 alterations also play a role in DNA damage repair and cell cycle control (review [4]). Cell culture experiments have shown that the RUNX1-RUNX1T1 fusion protein interferes with DNA repair, mainly by depressing genes related to baseexcision repair and double-strand break repair (mainly homologous recombination). Mutant, and thus inactivated, RUNX1 has negative effects on the Fanconi anemia (FA)/BReast CAncer (BRCA) susceptibility pathway.
RUNX1 mutations may also foster the survival of mutated hematopoietic stem cells by reducing p53 protein levels and activity as mutant RUNX1 can no longer act as a cofactor of p53.

In $\mathrm{t}(8 ; 21)$-positive AML, the mechanism of PAX5 (and, consecutively, CD79a) expression has already been uncovered. Ray et al. [15] demonstrated, in cell line models, that PAX5 is not a direct target of the chimeric RUNX1-RUNX1T1 but is activated by aberrant mitogenactivated protein kinase (MAPK) signaling. This is a result of the chimeric protein's action, leading to a dissociation of the Polycomb complex. As some, but not all, patients with $\mathrm{t}(8 ; 21)$ have CKIT mutations, which can explain the activation of MAPK signaling as well, another putative mode of action has been assumed by the induction or repression of various miRNAs by mutant/altered RUNX1 [16]. Finally, chimeric RUNX1-RUNX1T1 proteins inactivate the myeloid master regulator transcription factor PU.1, disturbing the cellular identity program 
and leading to differentiation stop, which may contribute to indel (B cell) lineage marker expression [17].

The aberrant $\mathrm{B}$ cell marker expression observed by us in MPO-negative or merely positive RUNX1 mutant AML is an important potential pitfall for the differential diagnosis of ALL, showing the significance of including FCM and genetic data in the integrative diagnosis of leukemias. FCM could render the diagnosis of AML versus ALL with greater accuracy, by relying on myelogenous markers such as CD33, which are only rarely part of the routine IHC panel of bone marrow biopsies because of weaker expression and a lack of blast specificity. Our observation suggests that, in addition to AML cases with $\mathrm{t}(8 ; 21)$ leading to RUNX1-inactivating RUNX1-RUNX1T1 fusions, deleterious point mutations of RUNX1 can, analogously to the former, promote the PAX5-mediated activation of $B$ cell marker expression in neoplastic myeloid blasts. It is important for diagnosticians to be aware of this.

\section{References}

1 Ichikawa M, Asai T, Saito T, Seo S, Yamazaki I, Yamagata T, et al. AML-1 is required for megakaryocytic maturation and lymphocytic differentiation, but not for maintenance of hematopoietic stem cells in adult hematopoiesis. Nat Med. 2004 Mar;10(3):299-304.

2 Mangan JK, Speck NA. RUNX1 mutations in clonal myeloid disorders: from conventional cytogenetics to next generation sequencing, a story 40 years in the making. Crit Rev Oncog. 2011;16(1-2):77-91.

3 Wildonger J, Mann RS. The t $(8 ; 21)$ translocation converts AML1 into a constitutive transcriptional repressor. Development. 2005 May;132(10):2263-72.

4 Bellissimo DC, Speck NA. RUNX1 Mutations in Inherited and Sporadic Leukemia. Front Cell Dev Biol. 2017 Dec;5:111.

5 Kozlov I, Beason K, Yu C, Hughson M. CD79a expression in acute myeloid leukemia $t(8 ; 21)$ and the importance of cytogenetics in the diagnosis of leukemias with immunophenotypic ambiguity. Cancer Genet Cytogenet. 2005 Nov;163(1):62-7.

6 Tiacci E, Pileri S, Orleth A, Pacini R, Tabarrini A, Frenguelli F, et al. PAX5 expression in acute leukemias: higher B-lineage specificity than CD79a and selective association with $\mathrm{t}(8$; 21)-acute myelogenous leukemia. Cancer Res. 2004 Oct;64(20):7399-404.
7 Ito Y. Oncogenic potential of the RUNX gene family: 'overview'. Oncogene. 2004 May; 23(24):4198-208.

8 Arber D, Brunning R, Le Beau M, Falini B, Vardiman J, Porwit A, et al. Acute myeloid leukaemia with recurrent genetic abnormalities. In: Swerdlow S, Campo E, Harris N, Jaffe E, Pileri S, Stein H, et al., editors. WHO Classification of Tumours of Haematopoietic and Lymphoid Tissues. Lyon: IARC; 2017. pp. 144-5.

9 Yamato G, Shiba N, Yoshida K, Hara Y, Shiraishi Y, Ohki K, et al. RUNX1 mutations in pediatric acute myeloid leukemia are associated with distinct genetic features and an inferior prognosis. Blood. 2018 May; 131(20): 2266-70.

10 Papaemmanuil E, Gerstung M, Bullinger L, Gaidzik VI, Paschka P, Roberts ND, et al. Genomic Classification and Prognosis in Acute Myeloid Leukemia. N Engl J Med. 2016 Jun; 374(23):2209-21.

11 Godley LA. Inherited predisposition to acute myeloid leukemia. Semin Hematol. 2014 Oct; 51(4):306-21.

12 Adams H, Liebisch P, Schmid P, Dirnhofer S, Tzankov A. Diagnostic utility of the B-cell lineage markers CD20, CD79a, PAX5, and CD19 in paraffin-embedded tissues from lymphoid neoplasms. Appl Immunohistochem $\mathrm{Mol}$ Morphol. 2009 Mar;17(2):96-101.
13 Morgan NV, Daly ME. Gene of the issue: RUNX1 mutations and inherited bleeding. Platelets. 2017 Mar;28(2):208-10.

14 Gaidzik VI, Teleanu V, Papaemmanuil E, Weber D, Paschka P, Hahn J, et al. RUNX1 mutations in acute myeloid leukemia are associated with distinct clinico-pathologic and genetic features. Leukemia. 2016 Nov;30(11): 2160-8.

15 Ray D, Kwon SY, Tagoh H, Heidenreich O, Ptasinska A, Bonifer C. Lineage-inappropriate PAX5 expression in $\mathrm{t}(8 ; 21)$ acute myeloid leukemia requires signaling-mediated abrogation of polycomb repression. Blood. 2013 Aug;122(5):759-69.

16 Zaidi SK, Dowdy CR, van Wijnen AJ, Lian JB, Raza A, Stein JL, et al. Altered Runx1 subnuclear targeting enhances myeloid cell proliferation and blocks differentiation by activating a miR-24/MKP-7/MAPK network. Cancer Res. 2009 Nov;69(21):8249-55.

17 Vangala RK, Heiss-Neumann MS, Rangatia JS, Singh SM, Schoch C, Tenen DG, et al. The myeloid master regulator transcription factor PU.1 is inactivated by AML1-ETO in $\mathrm{t}(8 ; 21)$ myeloid leukemia. Blood. 2003 Jan;101(1): 270-7. 